\title{
Global DC Closed Orbit Correction Experiments on the NSLS X-ray. Ring and SPEAR*
}

\author{
Y. Chung, G. Decker, and K. Evans, Jr. \\ Argonne National Laboratory, Argonne, IL 60439 \\ J. Safranek, I. So, and Y. Tang \\ Brookhaven National Laboratory, Upton, NY 11973 \\ W. J. Corbett and R. Hettel \\ Stanford Linear Accelerator Center, Stanford, CA 94305
}

\begin{abstract}
The global closed orbit correction experiments conducted on the NSLS X-ray ring and the SPEAR using the technique of singular value decomposition (SVD) are presented. The beam response matrix, defined as beam motion at beam position monitor (BPM) locations per unit kick by corrector magnets, was measured and then analyzed using SVD. The BPMs and correctors are reconfigured into "transformed" BPMs (t-BPMs) and "transformed" correctors (t-correctors), with each $t-B P M$ coupled to at most one $t$-corrector and vice versa for orbit correction. The decoupled $t-B P M s$ are used to estimate the limit on orbit correction, while the decoupled tcorrectors are used to optimize the corrector strengths. As a result, the vertical r.m.s. orbit error at the BPM locations was reduced from $208 \mu \mathrm{m}$ to $61 \mu \mathrm{m}$ about an arbitrary reference orbit in the NSLS X-ray ring. In SPEAR, the vertical closed orbit was brought to the BPM centers at the selected BPM locations with an r.m.s. error of $215 \mu \mathrm{m}$ reduced from the initial $780 \mu \mathrm{m}$.
\end{abstract}

\section{INTRODUCTION}

The third generation synchrotron light sources, of which the Advanced Photon Source (APS) is one, are characterized by low emittance of the charged particle beams and high brightness of the photon beams radiated from insertion devices. Transverse stability of the particle beams is a crucial element in achieving these goals and the APS will implement extensive beam position feedback systems, which include 320 corrector magnets, 360 positron beam position monitors (BPMs) distributed around the storage ring, miniature BPMs for insertion device beamlines, and photon beam position monitors in the front end of $\mathrm{X}$-ray beamlines.

The beam position feedback systems can largely be divided into the global and local feedback systems according to the extent of correction, and the DC and AC feedback systems according to the bandwidth of correction.

In this paper, we will present the results of global $D C$ beam position feedback experiments conducted on the $\mathrm{X}$-ray ring of

*Work supported by the U.S. Department of Energy, Office of Basic Energy Sciences, under Contract No. W-31-109-ENG-38. the National Synchrotron Light Source (NSLS) and on SPEAR at Stanford Synchrotron Radiation Laboratory (SSRL). Integral control with full correction was used, and the technique of singular value decomposition (SVD) was used to invert the response matrix.

The rest of this paper will consist of a brief review of the theory of SVD applied to closed orbit correction in Section II, analysis of the response matrices in Section III, and presentation of the measurement results in Section IV.

\section{THEORY}

The mathematical formulation of the SVD of matrices is broadly available in the literature [1-4] and its successful application to closed orbit correction at Aladdin, SRC, was reported in Ref. [5]. A theoretical treatment and physical interpretation of the technique for closed orbit correction is presented in Ref. [6], including minimization of orbit error and optimization of corrector strengths. In this section, we will overview the SVD formalism in order to introduce the terminology used in following sections.

Suppose that there are M BPMs and N correctors used for closed orbit correction in the storage ring. Assuming linearity, the response matrix $R_{i j}$ is defined as the beam motion at the $i$ th BPM per unit angle of kick at the $j$-th corrector. The orbit change $\Delta x$ due to the corrector strength change $\Delta \theta$ is then given by

$$
\Delta \mathbf{x}=\mathbf{R} \cdot \Delta \boldsymbol{\theta} .
$$

Using SVD, we write the response matrix $\mathbf{R}$ as [4]

$$
\mathbf{R}=\mathbf{U} \cdot \mathbf{W} \cdot \mathbf{V}^{\mathrm{T}},
$$

where $\mathbf{U}$ is an $\mathrm{M} \times \mathrm{M}$ unitary matrix $\left(\mathbf{U}^{\mathrm{T}} \cdot \mathbf{U}=\mathbf{U} \cdot \mathbf{U}^{\mathrm{T}}=1\right)$, $\mathrm{W}$ is an $\mathrm{M} \times \mathrm{N}$ diagonal matrix with positive or zero elements, and $\mathrm{V}$ is an $\mathrm{N} \times \mathrm{N}$ unitary matrix $\left(\mathrm{V}^{\mathrm{T}} \cdot \mathrm{V}=\mathrm{V} \cdot \mathrm{V}^{\mathrm{T}}=\mathbf{1}\right)$.

From Eqs. (1) and (2) and the unitary property of $U$ and $V$, we have

$$
\Delta \mathbf{x}^{\mathbf{t}}=\mathbf{W} \cdot \Delta \boldsymbol{\theta}^{\mathrm{t}},
$$

\section{MASTER}

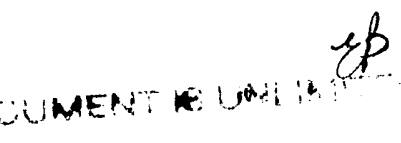

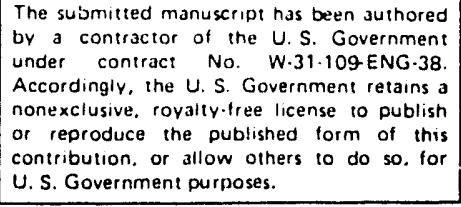


where $\Delta x^{\mathrm{t}}=\mathrm{U}^{\mathrm{T}} \cdot \Delta \mathrm{x}$ and $\Delta \theta^{\mathrm{t}}=\mathrm{V}^{\mathrm{T}} \cdot \Delta \theta$ in the transformed (t-) BPM and corrector spaces. The matrix $\mathbf{W}$ is given by

$$
\mathrm{W}_{\mathrm{ij}}=\mathrm{w}_{\min (\mathrm{ij})} \delta_{\mathrm{ij}} \text {, }
$$

where the diagonal elements $w_{n}(\geq 0,1 \leq n \leq \min (M, N))$ are called eigenvalues. Each represents the coupling efficiency of the correction channel of a pair consisting of a t-BPM and a tcorrector. The matrix $\mathbf{R}$ is singular if any of the eigenvalues is zero.

Given an orbit perturbation $\Delta \mathbf{x}$, the necessary corrector strength $\Delta \theta$ is the product of the inverse matrix $\mathbf{R}_{\mathrm{inv}}$ given by

$$
\mathbf{R}_{\mathrm{inv}}=\mathbf{V} \cdot \mathbf{W}_{\mathrm{inv}} \cdot \mathbf{U}^{\mathbf{T}}
$$

and $\Delta \mathbf{x}, \mathbf{W}_{\mathrm{inv}}$ is a diagonal matrix of dimension $\mathrm{N} \times \mathrm{M}$ and the elements are given by

$$
W_{i n v, i j}=q_{\min (i, j)} \delta_{i j},
$$

where

$$
q_{n}=\left\{\begin{array}{ll}
0, & w_{n} \leq \varepsilon w_{\max } \\
\frac{1}{w_{n}} . & \text { otherwise }
\end{array} \quad(1 \leq n \leq \min (M, N))\right.
$$

$\varepsilon$ is the singularity rejection parameter in the range $[0,1]$. For a given matrix $R$, we define $\varepsilon_{\mathrm{m}}(\mathbf{R})$ as

$$
\varepsilon_{m}(\mathbf{R})=\max \left\{\varepsilon \mid w_{n}>\varepsilon w_{\max } \text { for all } w_{n} \neq 0\right\} .
$$

That is, $\varepsilon_{\mathrm{m}}$ is the largest possible value for $\varepsilon$ in order to retain all non-zero eigenvalues.

\section{ANALYSIS OF RESPONSE MATRICES}

In this section, we will analyze the response matrices of the NSLS X-ray ring and SPEAR in the vertical plane.

\section{A. NSLS X-ray Ring}

We used all of the $48(\mathrm{M})$ BPMs and $39(\mathrm{~N})$ correctors available for orbit correction. The nominal vertical tune of the

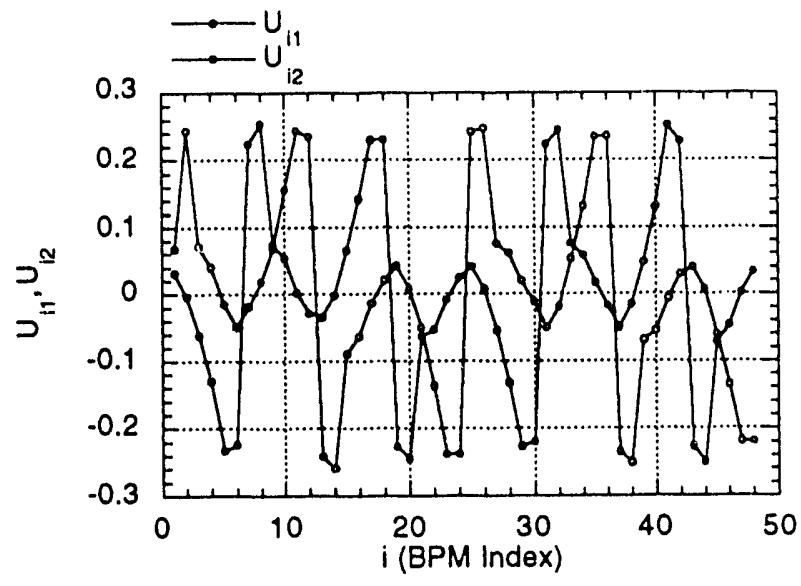

Fig. 1: The basis vectors $U_{i 1}$ and $U_{i 2}$ of the NSLS $X$-ray ring for the most strongly coupled channels.

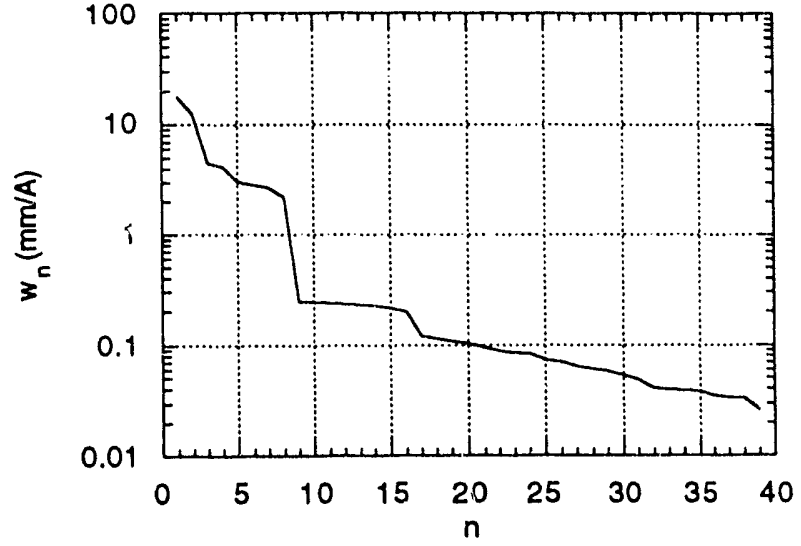

Fig. 2: Plot of the eigenvalues of the response matrix for the NSLS X-ray ring. $\varepsilon_{\mathrm{m}}=1.47 \times 10^{-3}$.

machine is $v_{\mathrm{v}}=6.2$. The response matrix was measured as the ratio of the beam motion in $\mathrm{mm}$ and the applied rurrent on the corrector magnets in amperes. The conversion to the angle is done using

$\Delta \theta(\mathrm{rad})= \begin{cases}4.20 \times 10^{-5} \Delta \mathrm{I}(\mathrm{A}), & \text { V8 correctors } \\ 6.87 \times 10^{-5} \Delta \mathrm{I}(\mathrm{A}) . & \text { others }\end{cases}$

Figure 1 shows the BPM basis vectors $\mathrm{U}_{\mathrm{i} 1}$ and $\mathrm{U}_{\mathrm{i} 2}(1 \leq \mathrm{i} \leq$ 48) for the most strongly coupled channels. These two vectors exhibit pseudo-periodicity of the integer tune 6 and are shifted in phase by approximately 90 degrees. This indicates that the two channels will mostly correct $m=6$ harmonic mode. The eigenvalues $w_{n}(1 \leq n \leq 39)$ are shown in Fig. 2. $\varepsilon_{m}$ of the response matrix is $1.47 \times 10^{-3}$.

Table 1 summarizes the results of simulation of orbit correction on the initial r.m.s. orbit error of $207.8 \mu \mathrm{m}$ using a different number $\left(n_{i}\right)$ of eigenvalues. The theoretical limit on the residual error after correction is $46.6 \mu \mathrm{m}$.

Table 1: Simulation of orbit correction in the vertical plane of NSLS X-ray ring using a different number $\left(n_{i}\right)$ of eigenvalues.

\begin{tabular}{|c|cccc|}
\hline $\mathrm{n}_{\mathrm{i}}$ & $\Delta \mathrm{I}_{\min }(\mathrm{A})$ & $\Delta \mathrm{I}_{\max }(\mathrm{A})$ & $\Delta \mathrm{I}_{\text {rms }}(\mathrm{A})$ & $\Delta \mathrm{y}_{\mathrm{rms}}(\mu \mathrm{m})$ \\
\hline 0 & 0.000 & 0.000 & 0.000 & 207.8 \\
2 & -0.010 & 0.011 & 0.007 & 183.2 \\
6 & -0.83 & 0.09 & 0.044 & 128.9 \\
10 & -1.01 & 0.13 & 0.30 & 108.8 \\
20 & -1.39 & 0.89 & 0.52 & 84.0 \\
30 & -2.90 & 1.81 & 1.01 & 68.9 \\
34 & -4.50 & 2.11 & 1.39 & 59.7 \\
36 & -4.88 & 3.03 & 1.65 & 52.2 \\
38 & -4.98 & 3.01 & 1.66 & 52.1 \\
39 & -5.40 & 3.78 & 1.93 & 46.6 \\
\hline
\end{tabular}

\section{B. SPEAR}

As in the case of the NSLS X-ray ring, all of the 26 BPMs and 30 correctors available for orbit correction were used. The nominal vertical tune of the machine is $v_{v}=6.72$. As shown in Fig. 3, the response matrix is nearly singular, and the weakest four channels $(n=23-26)$ may not be usable unless 
the power supplies are very robust. $\varepsilon_{\mathrm{m}}$ of the response matrix is $4.43 \times 10^{-4}$.

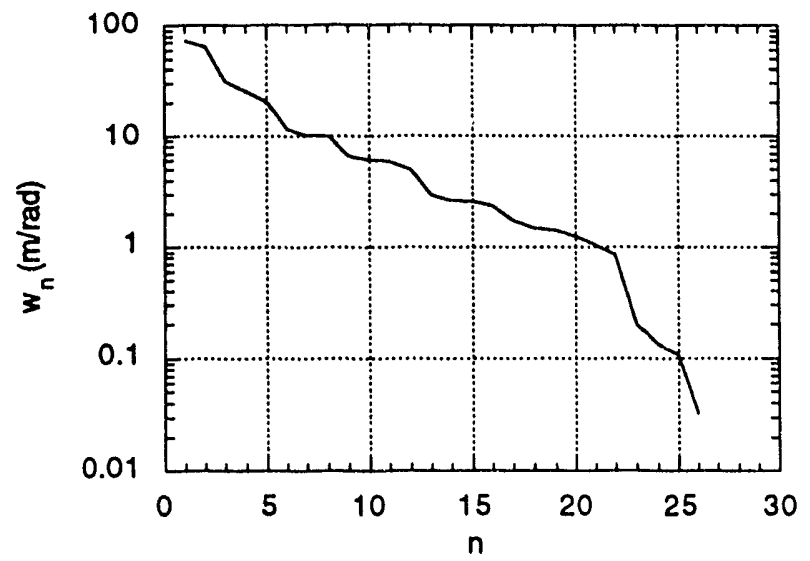

Fig. 3: Plot of the eigenvalues of the response matrix for SPEAR. $\varepsilon_{\mathrm{m}}=4.43 \times 10^{-4}$.

\section{MEASUREMENT RESULTS}

Figure 4 shows the result of orbit correction using 35 eigenvalues on the NSLS X-ray ring. The initial r.m.s. orbit error was $138 \mu \mathrm{m}$, which was reduced to $61 \mu \mathrm{m}$ after correction. A few iterations were necessary before the r.m.s. error settled down to this value, possibly due to changes in machine condition and error in the response matrix measurement. The corrector current changes ranged from $-4.56 \mathrm{~A}$ to $2.45 \mathrm{~A}$, with the r.m.s. value of $1.47 \mathrm{~A}$. Some of the corrector power supplies got close to, but did not reach, saturation at the maximum current of $10 \mathrm{~A}$. Including more eigenvalues would trip off some of the power supplies and was not tried.

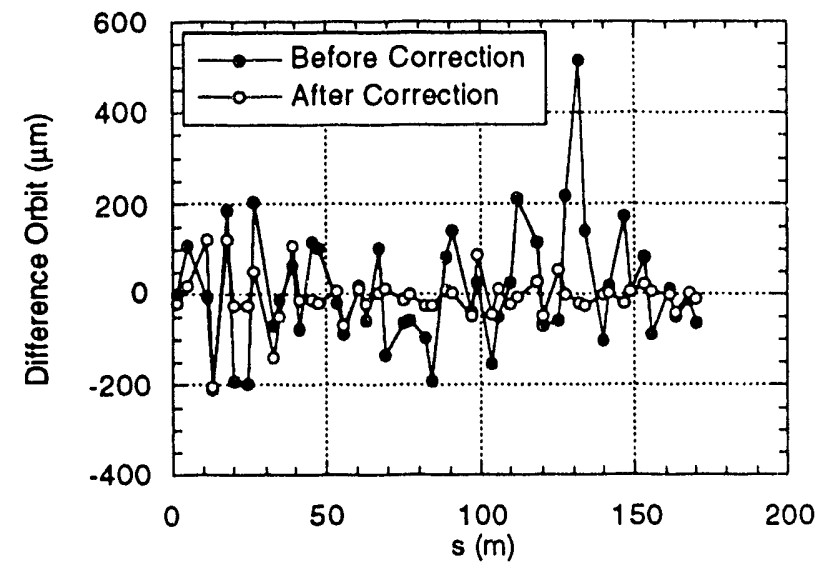

Fig. 4: DC Global orbit correction on the NSLS X-ray ring. The r.m.s. orbit error relative to the reference orbit is $137 \mu \mathrm{m}$ and $61 \mu \mathrm{m}$ before and after correction, respectively. Thirtyfive eigenvalues out of 39 were used.

In the case of SPEAR, we tried to bring the orbit as close as possible to the electrical centers of the BPMs, which were used as the reference for orbit correction. After each correction, the RESOLVE [7] computer program was used to fit the measured orbit to the machine model. This enabled us to locate some of the BPMs with significant of fsets and remove them for orbit correction. Using this procedure, 9 out of 26 were removed, and the result is shown in Fig. 5. The r.m.s. orbit error was reduced from $780 \mu \mathrm{m}$ to $215 \mu \mathrm{m}$ at the locations of the 17 remaining BPMs using 15 eigenvalues. Two channels had to be decoupled to avoid power supply saturation resulting from the misalignment of a BPM with the center of an adjacent quadrupole. This led to the kink in the orbit between the 9th and 10th BPMs.

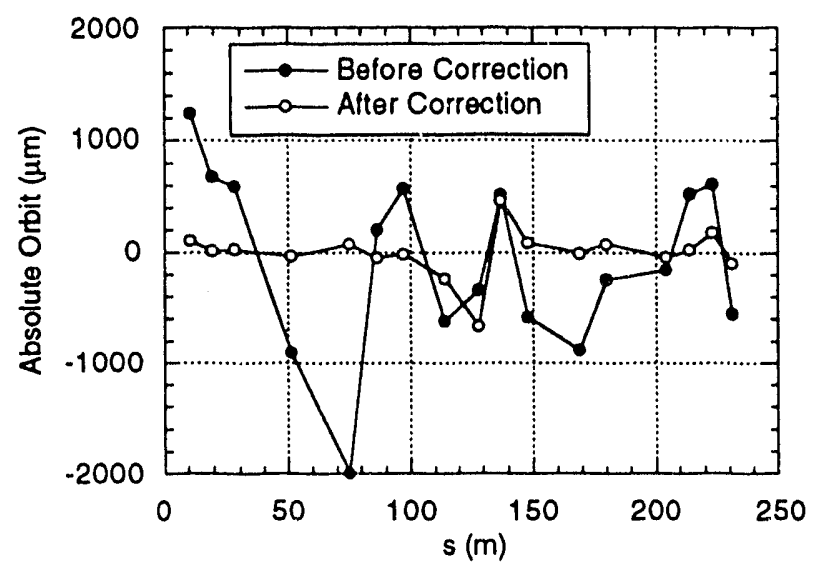

Fig. 5: DC global absolute orbit correction at SPEAR, SSRL. The r.m.s. orbit error relative to the vacuum chamber center is $780 \mu \mathrm{m}$ and $215 \mu \mathrm{m}$ before and after correction, respectively. Fifteen eigenvalues out of 17 were used.

\section{REFERENCES}

[1] G. H. Golub and C. Reinsch, "Singular Value Decomposition and Least Squares Solutions," Numer. Math. 14, pp. 403-420,1970, and references therein. Also in J. H. Wilkinson and C. Reinsch, Linear Algebra, vol. Il of Handbook for Automatic Computation, Springer-Verlag, New York, 1971.

[2] G. E. Forsythe, M. A. Malcolm, and C. B. Moler, Computer Methods for Mathematical Computations, Prentice-Hall, Englewond Cliffs, N.J., 1977.

[3] J. J. Dongarra, et al., LINPACK User's Guide, Chapter 11. Society for Industrial and Applied Mathematics, Philadelphia, 1979.

[4] MATLAB ${ }^{\text {TM }}$ User's Guide, The Mathworks, Inc., p. 3$178,1990$.

[5] K. J. Kleman, "Beam Diagnostics and Control at Aladdin," Nuclear Inst. and Meth., A266, p. 172, 1988.

[6] Y. Chung, G. Decker, and K. Evans, Jr., "Closed Orbit Correction Using Singular Value Decomposition of the Response Matrix," these proceedings.

[7] W. J. Corbett, M. J. Lee, and Y. Zambre, "Automatic Beamline Calibration Procedures," Proceedings of the 3rd European Particle Accelerator Conference, Berlin, p. 753, 1992. Cf. M. J. Lee for information on RESOLVE. 

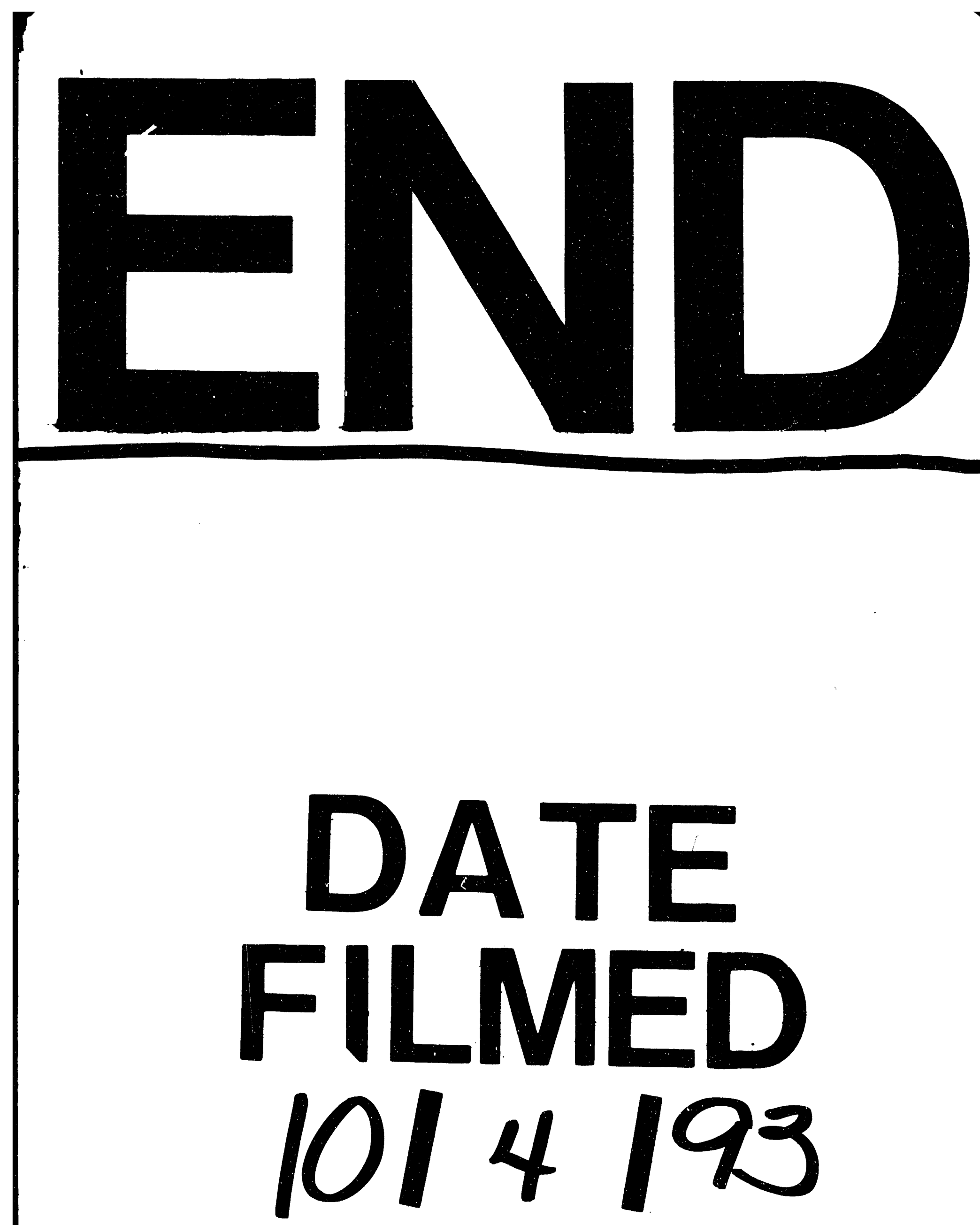
